Findings will help develop criteria to objectively and proactively define complex patients and improve care through greater team collaboration.

\section{PERSON CENTRED CULTURE IN A HOSPICE: MYTH OR REALITY?}

Erna Haraldsdottir, Kim Donaldson, Irene Barclay, Anna Lloyd, Brendan McCormac. St. Columba's Hospice, Queen Margaret University

\subsection{6/bmjspcare-2018-ASPabstracts. 148}

Background Person centred care is inherently integrated into palliative care as one of its cornerstones. Having developed from a pioneering grass route movement to an established organisational structure the culture of palliative care has become increasingly influenced by routine and the need for standardised practice. This has challenged the capacity to foster creative, flexible and dynamic approaches to care that put the person at the centre. McCormack and McCance's Person Centred Practice Framework (2017) is a theoretical framework that addresses such limitations. Using this framework as an underpinning theory in our research we aimed to assess and develop a person centred culture in a hospice.

Aim To evaluate and develop a person centred culture in a hospice setting.

Method Participatory action research using a cooperative inquiry approach involving key stakeholders within the hospice.

- Research Group established with staff members being coresearchers

- Values and beliefs exercise around the meaning of person centred care across the hospice.

- Baseline data collection including observations and interviews to identify the existing culture.

- Analysis of base line data and mapping against the Person Centred Practice Framework.

- Identifying key areas for further exploration and development.

- Development and implementation of an action plan.

Results

- Staff relationships and communication, identified as a key area needing further exploration.

- A fourth generation evaluation (Guba and Lincoln 1989) workshop with all teams in the hospice highlighted a lack of time for staff to reflect on practice and engage in meaningful relationships with each other and thus neglecting the positive impact of effective teamwork.

- Action plan, including development of stronger team relationships was implemented.

Conclusion McCormack and McCance (2017) consider the existence of 'healthful relationships' as central to a person-centred culture. This research highlights a practical approach for teams to explore their own micro level structures in order to develop healthful relationships in teams.

\section{2 \\ THE BIGGART PROJECT: COLLABORATION BETWEEN HOSPICE AND GERIATRIC COMMUNITY HOSPITAL TOWARDS SHARED LEARNING}

Cat Killin, Jillian Nicoll, Meriel Conn, Josaleen Connolly, Fiona Ewart, James Mack, Morag Thomson, Morag Cahir, Rachel Pugh, Shona Hynd. The Ayrshire Hospice, The Biggart Hospital, NHS Ayrshire and Arran

10.1136/bmjspcare-2018-ASPabstracts. 149

Background The increasing need for end of life care in older people meant Biggart Hospital admitting more terminally ill patients and the Ayrshire Hospice managing more complex co-morbidity. To deliver best palliative care, shared learning was needed.

Method Key personnel from both sites met bimonthly over an 18 month period. Challenges were identified and strategy determined, nurses' learning needs having been evaluated by survey. Senior nursing and medical staff visited the hospice as clinical observers. Palliative care ANP attended Biggart weekly. A bespoke teaching programme was delivered to Biggart with four different sessions provided for nursing staff. Teaching sessions were delivered by hospice speciality doctor to geriatrics trainees. Patients and carers/family were surveyed about their care on completion.

Results Before the teaching programme, 26/30 respondents $(87 \%)$ wished for further palliative care education. Key concerns included pain and symptom management, and a need for a key point of regular contact for advice. After the programme a second staff survey was conducted. 13/17 respondents were keen for more palliative care education. Their main concerns were pain (10), end of life care (10), advance planning and DNACPR (6), care of relatives (1). Of note, this was not the same group that received education or who replied to the first survey. Programme attendees who gave feedback reported increased awareness of palliative care. Following the training programme, relatives $(n=10)$ felt care in Biggart was 'always good' (10/10), pain/symptoms 'always' or 'usually' well managed $(5 / 10 ; 5 / 10$ respectively); sufficiently supportive both emotionally (8/10) and spiritually (4/10).

Conclusions Joint working improved relationships, skills and confidence. ANP visits consolidated this new approach. Education programme was valued by attendees albeit numbers were small.

Education programmes need learner and management commitment to support attendance. Pain, spiritual support and care of relatives remain key areas for ongoing education.

\section{ADDRESSING PALLIATIVE CARE STAFF FEARS THAT DISCUSSING CORNEA DONATION IS DISTRESSING FOR PEOPLE}

Sarah Mollart, Emma Tregenna. St. Nicholas Hospice Care

10.1136/bmjspcare-2018-ASPabstracts. 150

Background Cornea disease causes blindness. Cornea transplants are highly successful, but limited by shortage of donated corneas. The UK annual shortfall is \%o¥500 corneas. 